\title{
Comentarios acerca de dos casos de embarazo ectópico a término
}

\author{
Eduardo Acosta Bendek (*)
}

Sólo tiene por objeto la publicación de estos dos casos, contribuir con los ya registrados anteriormente al enriquecimiento ce nuestra literatura médica nacional, y asi contar con una estadística propia. La incidencia de los embarazos ectópicos a término es muy baja. En la Maternidad "Leopoldina de Santos", del Hospital de Barranquilla, en el lapso de seis años de 1951 a 1956, la incidencia de los embarazos ectópicos sobre 22.858 partos fue de $1: 142$ distribuídos así ectópicos rotos 90 , intactos 65 , ovári$\cos 3$, abdominales 2 , uno de los cuales a término, y tubo_intersticial a término uno; lo que arroja una incidencia de embarazos abdominales de $1: 11,429$; ectópicos a término abdominal y tubointersticial de $1: 22.858$.

Los sintomas y signos de los embarazos ectópicos son muy variados y complejos, y su diagnóstico reposa más que todo en una buena historia clínica; diagnóstico que debe hacerse antes del accidente, ya que no se nos escapa el potencial peligro que encierra para la vida humana este cuadro clínico; y haciendo nuestra la frase diríamos: "es menos ofensiva una laparotomía por exceso de celo que una muerte por negligencia”.

\section{Casos reportados}

Número 1. Paciente de cuarenta y un años, de raza mestiza, natural de Barranquilla, con antecedentes familiares sin importancia. Personales enfermedades de la infancia: ginecológicos, menarquia a los catorce años, ciclo $30 / 4$; obstétricos, grávida II, para I; un parto hace 17 años espontáneo y a término, atendido en su casa; 3 meses después del parto presentó un cuadro clínico de pelviperitonitis. Ultima regla, 28 de enero de 1956.

(*) Médico Jefe del Servicio de Maternidad "Leopoldina de Santos". Barranquilla. 
La paciente ingresa al Servicio de Maternidad el día 20 de noviembre del mismo año, por dejar de percibir los movimientos fetales desde hace 28 días; informa que a los 3 meses de amenorrea presentó dolores difusos abdominales que calmaron espontáneamente, y que se repitieron de una manera cíclica durante todo el embarazo. También registró hemorragia escasa por vagina mensualmente; en el mes de octubre presentó un falso trabajo de parto acompañado de una crisis dolorosa abdominal aguda, y desde ese momento no volvió a sentir los movimientos cel feto. Al examen obstétrico se encontró un abdomen globuloso, con $28 \mathrm{~cm}$. de altura uterina, y de consistencia dura que dificultó precisar la presentación. Ruidos fetales negativos, senos secretantes, pigmentaciones en la piel, órganos genitales externos normales, se observó un prolapso de cuello, orificio externo cerrado y de consistencia dura, útero haciendo parte de la tumefacción abdominal que se creía ser matriz; tensión arterial 120 $\mathrm{x} 75$, pulso 82, temperatura normal, aparato respiratorio y cardiovascular normal. Se hizo el diagnóstico de embarazo prolongado, feto muerto y retenido.

Evolución: Como no se presentó espontáneamente el trabajo de parto, se procedió a hacer la inducción médica con foliculina, a la aosis de $5 \mathrm{mg}$. diarios intramuscular durante 10 días, seguido de una solución de Pitocín al 1 por mil con resultados negativos; ocho días más tarde se repitió el mismo tratamiento sin respuesta alguna. Se hizo un estudio radiográfico que reveló un feto único con signos radiológicos de muerte fetal. En vista de los fracasos anteriores se hizo la inyección intra-amniótica de $30 \mathrm{~cm}$. de suero de mujer parturienta serológicamente negativa; durante esta maniobra no se observó a la aspiración presencia del líquido amniótico, por lo que se pensó que se trataba de reabsorción de éste, como sucede en los embarazos con feto muerto de evolución prolongada; este procedimiento inductivo también fracasó. Posteriormente de la inducción intraamniótica la enferma hizo un cuadro peritoneal con síntomas de pseudo oclu. sión intestinal que cedieron al tratamiento médico con antibióticos y succión gástrica permanente. Simultáneamente se registró un aumento de la masa abdominal con un enflaquecimiento progresivo de la enferma. Se intentó nueva inducción médica de la enferma haciendo un taponamiento cervical, combinado con solución pitocinada al 1 por mil por vía intravenosa y un enema jabonoso; con resultado también negativo. 
En vista de los fracasos obtenidos con el tratamiento médico inductivo, se resolvió hacer una cesárea por vía abdominal, desechando la vía vaginal por las consideraciones que se tuvieron de feto grande, cuello largo y duro, que dificultarían la extracción del feto por esta vía. Se hizo la paratomía con el diagnóstico de feto muerto in útero, retenido, gasometria y distocia cervical irreductible.

Descripción operatoria: Se hizo laparatomía mediana infraumbilical observándose congestión exagerada vascular de la pared abdominal, y peritoneo grueso y congestivo. Descubierta la cavidad abdominal se encontró un órgano muscular, cuyo aspec. to macroscópico reveló un proceso degenerativo de sus capas musculares, y su forma y consistencia como de un útero grávido, adherido al peritoneo parietal, epiplón y asas intestinales, observándose un proceso de peritonitis plástica; este órgano que a primera vista parecía ser útero, se abrió como tal, comprobándose que se trataba de la trompa derecha; la matriz se encontró rechazada a la izquierda de la pelvis, de consistencia, tamaño y forma aparentemente normales. Al abrir la trompa se encontró una colección purulenta de unos $300 \mathrm{~cm}$., y se hizo extracción de in feto muerto macerado (Figura número 1), que pesó $3.200 \mathrm{gm}$. y de aspecto aparentemente normal. La placenta se encontró implantada en la porción intersticial de la trompa y en estado de maceración. La trompa se liberó de todas las adherencias de intestino delgado, grueso y epiplón hasta llevar a cabo su extripación completa. Se conservó útero y anexo izquierdo que se encontrabian aparentemente normales (Figura número 2). Luego se peritonizó toda la región cruenta y se cerró la pared abdomi. nal en cuatro planos según la técnica acostumbrada; el post-operatorio fue normal; la paciente salió del Servicio en buenas condiciones el doceavo día de operada.

Número 2. Paciente' de treinta y tres años, natural de Bogotá, de raza blanca, ingresó al Servicio de Maternidad en un estado de excitación psico-motora que evolucionó rápidamente a un estado de obnubilación mental y sopor que dificultó obtener datos inmediatos sobre sus antecedentes. Al examen físico se en. contró: enferma en gran estado de deshidratación, edemas intensos de miembros inferiores, abdomen distendido y doloroso, temperatura de $380^{\circ} \mathrm{C}$., tensión arterial de $140 \times 90$, pulso blando filiforme, frecuencia de 140 por minuto, palidez acentuada de tegumentos y mucosa, lengua saburral y seca, sensorio embotado. Aparato circulatorio, taquicardia acentuada, ruidos cardíacos 


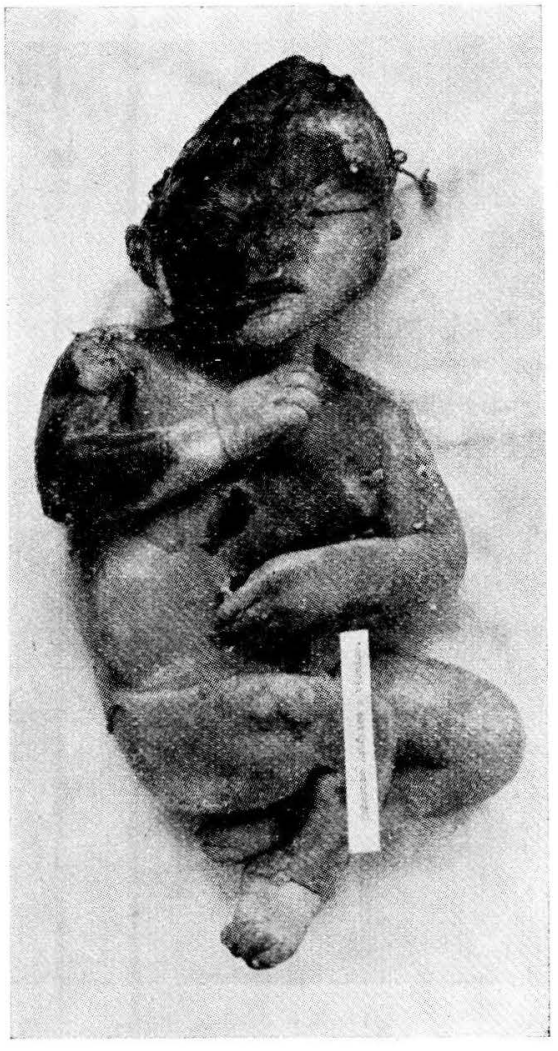

FIGURA NUMERO 1.-Feto muerto y macerado. Caso número 1.

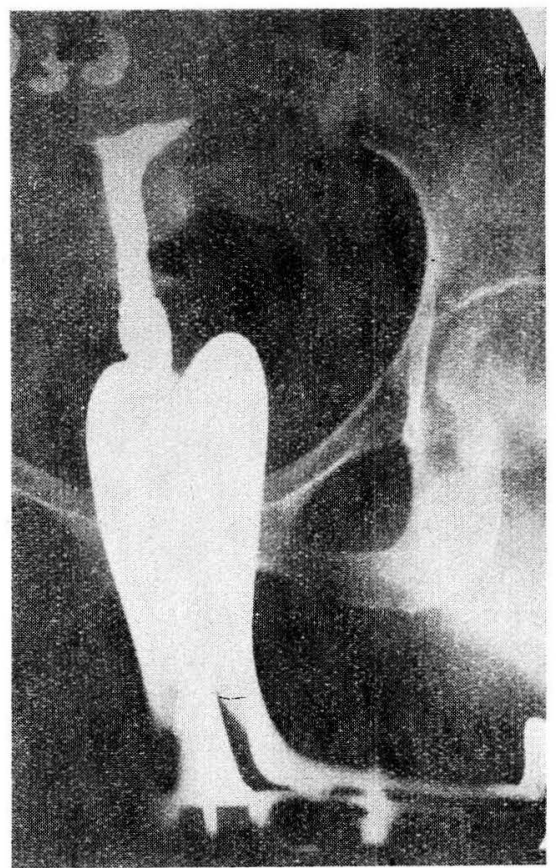

FIGURA NUMERO 2.-Historosalpingografía practicada a los 60 días de operada.

levemente velados; aparato respiratorio, se encontró polipnea 31 por minuto. Al examen obstétrico se encontró: abdomen globuJoso; con circulación colateral supraumbilical, aumentado de tamaño, exquisitamente doloroso y distendido que dificultó la palpación y apreciación de partes fetales; al tacto vaginal se observó genitales externos normales; en vagina se encontró en su tercio superior un tabique resistente en situación transversal y en forma de media luna que permitió pasar dos dedos en su parte superior y tocar un nuevo orificio de entrada de unos $5 \mathrm{~cm}$. de lar. go a través del cual se tocaba el polo cefálico separado por una delgada membrana, orificio que se consideró como una ruptura del segmento uterino. El cervix no se pudo localizar al tacto, y al 
sxamen con el especulum tampoco fue posible verlo, solamente se observó la presencia del tabique anteriormente ya descrito.

En vista de las condiciones anteriores de la enferma se procedió practicar cesárea abdominal con el diagnóstico de feto muerto, ruptura del segmento uterino y distocia de partes blandas.

Los datos que a continuación transcribo fueron obtenidos de la paciente al sexto día del post-operatorio. Antecedentes hereditarios y familiares sin importancia; patológicos, enfermedades propias de la infancia; apendicectomía y amigdalectomía hacía 15 o 10 años respectivamente; ginecológicos, menarquia a Jos quinec años, ciclo menstrual normal, tipo 30/4; obstétricos, un embarazo con parto normal a término hacía 13 años, grávida II. para I, última menstruación 8 de julio de 1956. Refiere la paciente, que un mes después de la amenorrea presentó un dolor súbito abdominal localizado en el epigastrio e hipocondrio derecho e izquierdo; este dolor con el reposo se convirtió en una sensación de peso y tensión dolorosa permanente durante tres dias acompañado de estreñimiento. Durante todo el período de la ges. tación la paciente experimentó en una forma cíclica (30 días) este dolor con duración de $72 \mathrm{~h}$., y que se presentaba en forma sistemática todos los 15 de cada mes; también registró vómitos constantes y frecuentes que se agrababan más en la época del dolor cíclico y presencia de una escasa secreción hemática oscura, espontánea e indolora a los 2 y 5 meses de embarazo; percibió ios movimientos fetales desde los cuatro y medio meses de gestación; el edema de los miembros inferiores se instaló desde los cinco meses; la paciente fue vista por varios médicos durante su estado, llegando al Servicio en las condiciones anteriormente ya anotadas, y después de 48 horas de haberse iniciado el trabajo de parto; refiere además la enferma que 4 días antes de ingresar al Servicio presentó un cólico abdominal agudo con pérdida de los movimientos fetales, asociado con distensión del abdomen y vómitos; el cuadro clínico fue cada día más grave hasta haber llega. co al estado en que se presentó al Servicio de Maternidad.

\section{Descripción operatoria}

Sa hizo incisión infraumbilical mediana y se abrió la pared abdominal según la técnica acostumbrada. Se observó un peritoneo parietal grueso, deslustrado y bien vascularizado adherido a útero, intestino delgado, grueso, y epiplón, se encontró un verdadero proceso de peritonitis plástica adhesiva. Ocupa todo el campo operatorio una gran masa llena de adherencia que se cre- 


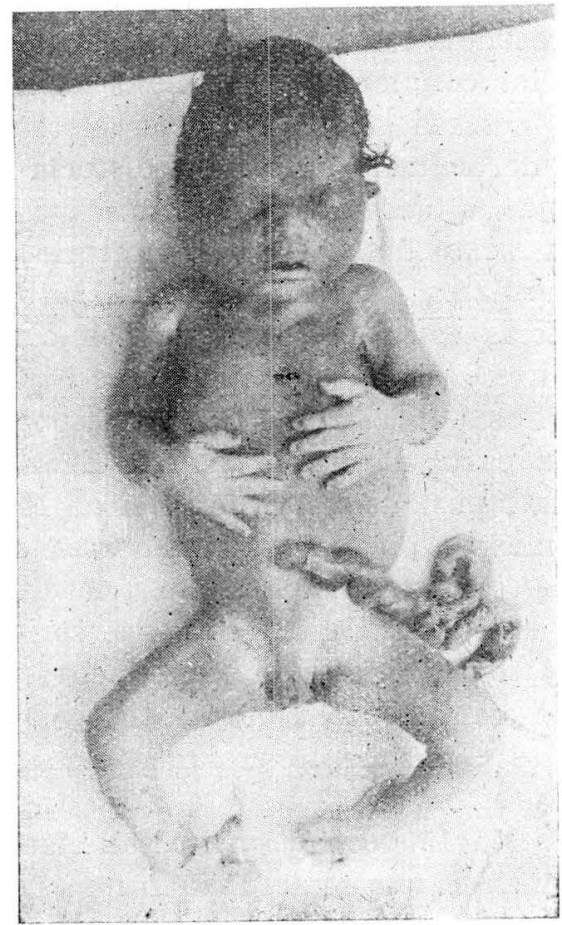

FIGURA NUMERO 3.-Feto a término del embarazo abdominal. Caso número 2.

yó ser el útero grávido a término; como fue difícil exteriorizar y hacer la liberación de las adherencias se procedió hacer una cesárea corporal, y con gran sorpresa nuestra encontramos un útero libre del cual salió una pequeña masa de caracteres semejantes a tejido placentario, que el examen histopatológico reveió ser tejido decidual; al continuar la exploración nos dimos cuenta que se trataba de un embarazo abdominal a término. En la cavidad peritoneal se observó la presencia de un líquido de color grisáceo y vísceras impregnadas de meconio, lo que nos hizo pensar en una ruptura del saco ovular más bien reciente. Luego se hizo la extracción de un feto muerto (Figura número 3), no macerado, con pie chapín bilateral, de sexo femenino, que pesó 2.700 gms., y que se encontraba detrás del útero y enclavado en el fondo de Saco de Douglas; la matriz formaba la pared anterior del saco ocular, y el embarazo le había imprimido un reblandecimiento tal que le permitió su distensión dando la impresión que tuvimos a primera vista de tratarse de un útero embarazado 
a término. El saco ovular ocupaba todo el fondo de saco de Dou. glas, en el cual se encontraba la cabeza fetal llenándolo por completo; la placenta estaba implantada en la región sacro lumbar (zona del promontorio), el extremo superior del saco membranoso sobrepasaba el ombligo unos cuatro centímetros. Debido al proceso de peritonitis plástica adhesiva fue muy difícil hacer la Extirpación completa del saco ovular, quedando restos de éste adheridos a las asas intestinales del delgado y grueso. Luego se procedió a practicar una histerectomía subtotal dadas las condiciones anatomo-patológicas del útero. La extracción de la placenta fue fácil, produciendo escasa hemorragia que se cohibió con compresas calientes. Se dejó un dren de Penrose a través del fondo de saco de Douglas que se retiró a las 48 horas. La pared abdominal se cerró en cuatro planos con catgut cromado número 2 , excepto el peritoneo que se usó simple 0 ; según la técnica acostumbrada. El post-operatorio fue normal y se le dió de alta a la paciente a los 15 días de operada.

El reporter histopatológico reveló ambos fetos completamen. te normales, por ser demasiado extenso dicho reporter sólo me limito a transcribir el diagnóstico final que fue el siguiente:

Caso número 1. Embarazo ectópico roto y feto a término macerado. Caso número 2. a) útero con reacción decidual del endometrio; b) embarazo tubárico de la trompa derecha; c) tejido decidual parcialmente necrótico y d) feto a término con pie chapín bilateral hemorragias petequiales en pulmones, pericardio, cerebro y cápsulas suprarrenales.-Firmado, doctor Arturo Alvarez $\mathbf{H}$.

\section{Comentarios}

En la mayoría de los casos de embarazo ectópico a término, el diagnóstico se' hace quirúrgicamente ya que la sintomatología es tan polimorfa y confusa que el diagnóstico clínico se hace muchas veces difícil, por no decir imposible. Llama la atención en los casos relatados la esterilidad secundaria por largos años, síntoma muy frecuente en la mayoría de los embarazos ectópicos, como también las crisis abdominales dolorosas asociadas a trastornos digestivos, las pérdidas sanguíneas por vagina de tipo cíclico, falsos trabajos de parto acompañados de dolor abdominal agudo, el fracaso de los tratamientos médicos inductivos, la fal. ta de reblandecimiento del cervix, y el embarazo prolongado con feto muerto y retenido. El estudio radiográfico sólo reveló la presencia de signos comunes a los de un embarazo corriente con 
feto muerto, no fueron muy claros los signos radiológicos que registra Brindeau en los casos de embarazos ectópicos.

Siempre que se presenten en pacientes embarazadas los síntomas anteriormente registrados es bueno pensar en la posibilidad de un embarazo ectópico.

El primero de nuestros casos fue un embarazo tubointersticial a término, localización muy rara, cuyo feto alcanzó un desarrollo y peso normales, sin anomalía congénita alguna, y cuya muerte se produjo más o menos a los 9 meses de gestación; y permaneciendo en la trompa hasta los 12 meses.

En el segundo caso según el repórter histopatológico se trata de un embarazo abdominal secundario, cuyo asiento primitivo fue la trompa derecha; gracias a este examen pudimos comprobar que se trataba de un embarazo abdominal secundario y no primario. Sin embargo hay algunos autores que niegan la existencia' de éste último, pero hoy ha sido ya un hecho comprobado que sí existe, como lo demuestran los casos registrados por Studdifortd en 1942 (1), y Arthur P. Martini (2), en marzo de 1955, y muchos otros para no citar más.

Sobre la conducta, soy de opinión que todo embarazo ectópico debe ser inmediatamente intervenido; haciendo la excepción en aquellos casos muy especiales, en los cuales la viabilidad fetal se aproxime casi al término, es decir, hasta la 36 semana de gestación, lo cual nos permite contemporizar un poco más; pero siempre preparados y alertas para cualquier emergencia que se presentare.

Con relación al tratamiento que se debe hacer de la placen. ta, soy partidario que ésta debe ser retirada si el feto tiene más de 72 horas de muerto y la extracción no es dificultosa; si en el momento de la intervención el feto se encontrare vivo, es aconsejable dejarla en su sitio de implantación ligando el cordón lo más cerca de la placenta.

El pronóstico de estas enfermas ha mejorado con los adelantos de la transfusión y presencia de los antibióticos. Aunque se ha culpado a estos del mayor porcentaje de embarazos ectópicos en los últimos años, concepto no de acuerdo con la realidad, ya que la culpa no es de los antibióticos, sino del mal uso y abuso que de ellos se hace.

\section{Resumen}

Se presentan dos casos de embarazo ectópico a término, uno tubo intersticial y otro abdominal secundario. El primero opera- 
da a los 12 meses, y el segundo a los 9; ambos con fetos muertos, uno de los cuales hacía 3 meses más o menos y el otro unos pocos días; el diagnóstico en los dos casos se hizo quirúrgicamente; ambas enfermas acusaban esterilidad secundaria de dieciocho y trece años. Las pacientes salieron del Servicio en buen estado general.

Doy las gracias al doctor Arturo Alvarez H., patólogo del Hospital de Barranquilla, por el estudio histopatológico de las pie. zas material de este estudio.

\section{BIBLIOGRAFIA}

1. STUDDIFORD, WILLIAM E.-Am. J. Obst. \& Gynec. 44: 487. 1942.

2. ARTHUR P. MARTINI.-"Early primary abdominal" (peritoneal) pregnancy. Am. J. Obst. \& Gynec. 73: 1139. 1957.

3. NAVAS ANGEL GUILLERMO.-"Casos curiosos de embarazo ectópico avanzado". Rev. Col. de Obst. y Gin. Vol. I, número 4, junio-julio. 1950.

4. GOMEZ PALACINO J. A.-“Un embarazo Tubo-intersticial a térmi1.0". Revista del Hospital San Juan de Dics. Vol. I, número 1. Enero-marzo. 1953.

5. RAMIREZ NERCHAN RAFAEL.-“Embarazo ectópico casi término". Revista Medicina y Cirugía. 1946.

6. SERPA ROBERTO.- "Embarazo extra-uterino". Revista de la Facultad Nacional de Medicina. 1940.

7. EARDLEY HOLLAND and ALECK BOURNE.-"British Gynaecological practice”. William Heinemann. Medical book. Ltda. London. 1955.

Barranquilla, julio 30 de 1957. 